\title{
Integrin $\beta 1$ Establishes Liver Microstructure and Modulates Transforming Growth Factor $\beta$ during Liver Development and Regeneration
}

\author{
Ryota Masuzaki, ${ }^{*}$ Kevin C. Ray, ${ }^{*}$ Joseph Roland, ${ }^{*}$ Roy Zent, ${ }^{\dagger \dagger}$ Youngmin A. Lee, ${ }^{*}$ and Seth J. Karp*
}

From the Section of Surgical Sciences* and the Department of Medicine, ${ }^{\dagger}$ Vanderbilt University School of Medicine, Nashville; and the Department of Medicine, ${ }^{\ddagger}$ Nashville Veterans Affairs Hospital, Nashville, Tennessee

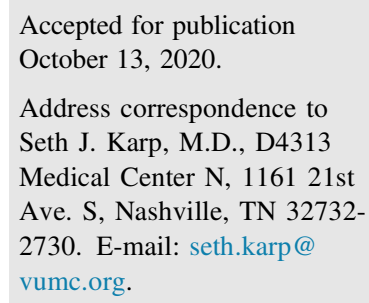

\begin{abstract}
A unique and complex microstructure underlies the diverse functions of the liver. Breakdown of this organization, as occurs in fibrosis and cirrhosis, impairs liver function and leads to disease. The role of integrin $\beta 1$ was examined both in establishing liver microstructure and recreating it after injury. Embryonic deletion of integrin $\beta 1$ in the liver disrupts the normal development of hepatocyte polarity, specification of cell-cell junctions, and canalicular formation. This in turn leads to the expression of transforming growth factor $\beta$ (TGF- $\beta$ ) and widespread fibrosis. Targeted deletion of integrin $\beta 1$ in adult hepatocytes prevents recreation of normal hepatocyte architecture after liver injury, with resultant fibrosis. In vitro, integrin $\beta 1$ is essential for canalicular formation and is needed to prevent stellate cell activation by modulating TGF- $\beta$. Taken together, these findings identify integrin $\beta 1$ as a key determinant of liver architecture with a critical role as a regulator of TGF- $\beta$ secretion. These results suggest that disrupting the hepatocyte-extracellular matrix interaction is sufficient to drive fibrosis. (Am J Pathol 2021, 191: 309-319; https://doi.org/10.1016/j.ajpath.2020.10.011)
\end{abstract}

The homogeneous appearance of the liver belies a complex microstructure essential for proper function. A fenestrated endothelium provides minimal resistance to blood flow to maintain low portal pressure and allows filtered plasma to bathe hepatocytes, permitting nutrient exchange and release of synthetic products. At the apical hepatocyte surface, a specialized canalicular network conveys bile out of the liver for secretion into the intestine.

Establishing precise microstructure is thus critical for liver function. The role of hepatocyte-extracellular matrix (ECM) interactions in the development of liver microstructure was therefore examined in the current study.

Integrins play an essential role in hepatocyte-ECM interactions. They are single-pass transmembrane receptors that function as adhesion molecules on the cell surface and mediate cell-matrix and cell-cell interactions. ${ }^{1}$ They exist on the hepatocyte surface as heterodimers of an $\alpha$ and $\beta$ subunit and contain a short cytoplasmic tail responsible for chemical signaling and physical force transduction through links to the actin cytoskeleton. ${ }^{2}$ Component subunits exhibit high selectivity in their interactions. In particular, integrin receptors on hepatocytes bind collagen I, laminin, and fibronectin. $^{3-}$

Integrin signaling involves a multiprotein complex that is recruited to and becomes associated with the intracellular portion of the protein. Although heterogeneous, signaling often involves focal adhesion kinase (FAK) as a platform for various phosphorylation events, including autophosphorylation, as well as signaling involving Src-family kinase, integrin-linked kinases, and paxillin. ${ }^{6-8}$ Principal integrin pairs expressed by hepatocytes are $\alpha 1 \beta 1, \alpha 5 \beta 1$, and a9 $\beta 1$., 10 The $\alpha 1 \beta 1$ heterodimer predominantly binds collagen IV, the $\alpha 5 \beta 1$ receptor binds fibronectin, and the $\alpha 9 \beta 1$ receptor binds to a non-RGD (Arg-Gly-Asp) site on tenascin. ${ }^{1,12}$ Data on

Supported by Veterans Administration grant 1I01BX002196-01 (R.Z.); NIH grants R01-DK069221 (R.Z.), R01-DK083187 (R.Z.), and 7R01DK081387 (S.J.K.); and Japan Society for the Promotion of ScienceJapan, Kakenhi grant JP20K08343 (R.M.).

Disclosures: None declared. 
specific roles for integrins in the liver are limited to a few reports. $\alpha 5 \beta 1$ integrins sense tauroursodeoxycholic acid and become active in response to cell swelling. ${ }^{13}$ Mice with transgene-mediated osteopontin expression in hepatocytes develop fibrotic livers. ${ }^{14}$ Disruption of integrin-linked kinase in hepatocytes leads to increased deposition of ECM. ${ }^{15}$ Integrin $\beta 1$ is essential for liver regeneration, but its effect on fibrosis has not been determined. ${ }^{16}$ In humans, hepatitis $\mathrm{C}$ is associated with increased expression of various integrins, including $\beta 1, \alpha 1, \alpha 5$, and $\alpha 6$, which reflect disease severity. ${ }^{17}$ Expression of integrin $\alpha 6$ occurs in a variety of chronic liver diseases. ${ }^{18}$

A critical role for integrins after injury is also suggested by the known role of ECM during injury response. ECM remodeling via matrix metalloproteinases (MMPs) and tissue inhibitors of metalloproteinases (TIMPs) is critical in recreating normal liver architecture. MMP-2, MMP-3, and MMP-14 are expressed by stellate cells during activation. ${ }^{19,20}$ MMP-9 is expressed in hepatocytes and Kupffer cells after setting of injury. ${ }^{21}$ TIMP-1 modulates liver MMPs, and both TIMP-1 and TIMP-2 are expressed in hepatocytes during injury. $22-24$

To determine how hepatocyte integrins help establish liver microstructure and re-establish it after liver injury, models were created to assess the consequences of hepatocyte-specific deletion of integrin $\beta 1$ from before birth and in the adult mice after injury. Hepatocyte size and shape, interactions between hepatocytes and other cells, and signaling pathways relevant to integrin signaling and fibrosis were investigated.

\section{Materials and Methods}

\section{Mice}

Vanderbilt University Medical Center Institutional Animal Use and Care Committee approved all experiments. Transgenic mice containing Cre recombinase under control of the albumin promoter were purchased from The Jackson Laboratory (Bar Harbor, ME). Integrin $\beta 1^{\text {flox/flox }}$ mice were provided by Dr. Elaine Fuchs (Howard Hughes Medical Institute, The Rockefeller University, New York, NY). ${ }^{25}$

\section{Virus Infection}

Adeno-associated virus 8 vector plasmid containing a hepatocyte-specific murine urinary protein promoter and cre-recombinase (AAV8-cre) was used for in vivo studies by injecting $7.5 \times 10^{10}$ DNase-resistant particles/mouse. ${ }^{26}$ Control virus contained enhanced green fluorescent protein (AAV8-eGFP).

\section{Creation of Hepatocyte-Specific Integrin $\beta 1$ Deletion}

Hepatocyte-specific deletion of integrin $\beta 1$ in utero was achieved using a transgenic strategy by crossing albumin-cre mice with integrin $\beta 1^{\text {flox/flox }}$ mice (hsd-itgb1-t). Control mice were cre-negative littermates. Hepatocytespecific deletion of integrin $\beta 1$ in adult mice was achieved by injecting integrin $\beta 1^{\text {flox/flox }}$ mice with AAV8-cre virus to delete integrin $\beta 1$ (hsd-itgb1-v). Control mice were injected with AAV8-eGFP.

\section{Partial Hepatectomy}

All surgeries were performed per the NIH recommendations contained in the Guide for the Care and Use of Laboratory Animals. ${ }^{27}$ Sixty $\mathrm{mg} / \mathrm{kg}$ ketamine (Hospira, Lake Forest, IL) and $7 \mathrm{mg} / \mathrm{kg}$ xylazine (Phoenix Pharmaceutics, Dayton, $\mathrm{OH})$ were used for anesthesia. An incision was made inferior to the xiphoid process, which was excised. Evisceration of the median and left lateral lobes allowed suture ligation and $60 \%$ hepatectomy. Sections were frozen in liquid nitrogen. Ten percent neutral buffered formalin was used as a fixative, and a portion of the tissue was preserved in RNALater (Qiagen, Hilden, Germany). Postoperative mortality was $<5 \%$ and independent of genotype.

\section{Histology and Immunohistochemistry}

Livers were fixed in $10 \%$ formalin, embedded, sectioned, and stained with hematoxylin and eosin or Sirius Red (Sirius Red \#365548; Sigma Aldrich, St. Louis, MO) as previously described. ${ }^{28}$ Weigert's hematoxylin was used as a nuclear stain. Immunohistochemistry was performed on paraffinembedded tissue according to standard protocols. The following were used: rabbit anti-human CK-19 (1:100; Novus, Centennial, CO), rabbit anti-mouse collagen I (1:200; Novus), rabbit polyclonal anti-claudin-3 (1:2000; Thermo Fisher Scientific, Waltham, MA), rabbit anti-mouse E-cadherin (1:500; Cell Signaling Technology, Danvers, MA), rabbit anti-human extracellular signal-regulated kinase 1/2 (ERK1/2; 1:500; Cell Signaling Technology), rabbit anti-human phospho-ERK1/2 (1:500; Cell Signaling Technology), rabbit anti-human phospho-ezrin/radixin/ moesin (1:500; Cell Signaling Technology), rabbit antihuman multidrug resistance transporter 1 (MDR1) (1:500, Abcam, Cambridge, MA), rabbit polyclonal anti- $\alpha$-smooth muscle actin (1:200; Abcam), and rabbit polyclonal anti- transforming growth factor- $\beta$ (TGF- $\beta 1$; 1:500; Abcam). TUNEL staining was performed after treating with proteinase $\mathrm{K}$ using the Click-iT TUNEL colorimetric IHC Detection Kit (Invitrogen, Waltham, MA).

\section{mRNA Isolation and Real-Time RT-PCR}

Thirty milligrams of tissue was used to purify total mRNA preserved in RNALater (RNeasy Mini Kit; Qiagen) as previously described. ${ }^{26}$ Briefly, mRNA was reverse transcribed to cDNA with a High Capacity cDNA Reverse Transcription Kit (Applied Biosystems, Foster City, CA). Real-time PCR used StepOnePlus (Applied Biosystems). 
cDNA template amplification was performed with TaqMan gene expression assays (Applied Biosystems) under manufacturer suggestions.

Gene expression levels were normalized to glyceraldehyde-3-phosphate dehydrogenase (GAPDH) by using the comparative CT method and analyzed with StepOne software version 2.1 (Applied Biosystems). TaqMan probe and primer sets (Applied Biosystems) were TGF- $\beta 1$ (Mm01178820_m1) and GAPDH (Mm99999915_g1). Preparation of JS1 cells involved washing and then RNA collection with an RNeasy Kit (Qiagen). cDNA was synthesized using Clontech (Takara, Japan). Real-time quantitative reserve transcription-PCR was performed with SYBR Green Mix (Bio-Rad, Hercules, CA).

\section{Western Blot Analysis}

Western blot analysis was performed as previously described. ${ }^{29}$ Briefly, $50 \mathrm{mg}$ of frozen tissue was homogenized in lysis buffer containing phosphatase and protease inhibitors. Samples were sonicated, clarified, and denatured. Bradford assay assessed protein concentration. SDS-PAGE in $4 \%$ to $12 \%$ or $12 \%$ NuPAGE Bis-Tris precast gels (Life Technologies, Waltham, MA) was used to separate proteins. In all cases, at least three mice were used at each time. Blots show representative images, and boxes designate separate photographs. Antibodies used were: TGF- $\beta 1$ (1:1000; Novus), ERK1/2 (1:1000; Cell Signaling), p-ERK1/2 (1:50; Cell Signaling), c-Jun N-terminal kinase (JNK) (1:1000; Cell Signaling), p-JNK (1:1000; Cell Signaling), p-protein kinase B (AKT) (1:2000; Cell Signaling), p-p38 (1:500; Cell Signaling), p-SMAD3 (1:500; Cell Signaling), TIMP-1 (1:250; Invitrogen), MMP13 (1:2000; Abcam), and $\alpha$-tubulin $(1: 10,000$; Cell Signaling). ImageJ software version 1.53a (NIH, Bethesda, MD; https://imagej.nih.gov/ij) was used for densitometry comparisons. Blots were stripped with Restore western blot stripping buffer (Thermo Scientific no. 21059) for 15 to 30 minutes at room temperature.

\section{Electron Microscopy}

Electron microscopy was performed by using standard protocols. Briefly, $2.5 \%$ glutaraldehyde in $0.1 \mathrm{~mol} / \mathrm{L}$ cacodylate buffer, $\mathrm{pH} 7.4$, was used to fix samples. Then, 0.1 $\mathrm{mol} / \mathrm{L}$ cacodylate buffer was used to wash samples, which were incubated in $1 \%$ osmium tetroxide followed by washing with $0.1 \mathrm{~mol} / \mathrm{L}$ cacodylate buffer. Samples were dehydrated through a graded ethanol series into propylene oxide. After infiltration with Epon 812 resin/propylene oxide, samples were embedded in epoxy resin.

Using a Leica Ultracut microtome (Leica Biosystems, Wetzlar, Germany), $500 \mathrm{~nm}$ to $1 \mu \mathrm{m}$ thick sections were cut; $1 \%$ toluidine blue was used for contrast, and 70 to $80 \mathrm{~nm}$ sections were laid on mesh copper grids and stained with $2 \%$ uranyl acetate and lead citrate. A Philips/FEI Tecnai
T12 electron microscope (FEI Co., Hillsboro, OR) was used for imaging.

\section{Primary Hepatocyte Sandwich Cultures}

Primary hepatocytes were cultured as previously described. ${ }^{29}$ Briefly, after anesthesia, the liver was perfused with pre-perfusion solution followed by collagenase (Gibco, Waltham, MA) via the portal vein. Mechanical dissociation in minimal essential medium was followed by filtering, washing, and plating. Sandwich configuration was achieved by overlaying rat tail collagen I (Corning, Corning, NY) followed by medium. Hepatocytes were harvested from approximately 12-week-old hsd-itgb1-v livers before the development of a phenotype.

\section{Primary Stellate Cell Cultures}

Primary stellate cells were purified using centrifugation on an $8 \%$ Nycodenz gradient (Axis-Shield Diagnostics, Dundee, Scotland, UK) as previously described. ${ }^{30}$

\section{Cell Culture}

JS1 cells were cultured in Dulbecco's modified Eagle's medium $/ 10 \%$ fetal bovine serum. ${ }^{31}$ For conditioned media experiments, JS1 cells were incubated for 48 hours with conditioned media from control or integrin $\beta 1$ knockout primary hepatocytes. Cells were washed, and RNA was then collected with the RNeasy Kit (Qiagen). cDNA was synthesized by using Clontech (Takara).

\section{Serum Chemistry}

Albumin, alanine transaminase, total bilirubin, total bile acids, and alkaline phosphatase were measured in serum using VetScan VS2 (Abaxis, Union City, CA). Serum retinol-binding protein 4 levels were measured by using an enzyme-linked immunosorbent assay kit (R\&D Systems, Minneapolis, MN).

\section{Statistical Analysis}

Comparisons used the two-tailed unpaired $t$-test. All statistical analysis included at least three independent samples each. $P<0.05$ was considered statistically significant. Error bars represent SEM.

\section{Results}

Integrin $\beta 1$ Is Essential for Microscopic Patterning of the Liver

Given the ubiquity and importance of cell-ECM communication during organ development, interactions between hepatocytes and ECM were hypothesized as required to produce normal gross and microscopic liver architecture. To investigate 
this theory, hsd-itgb1-t mice were generated by crossing floxed integrin $\beta 1$ mice with albumin-cre recombinase transgenic mice. The albumin promoter drives cre in the liver (hepatoblasts) beginning at embryonic day 14.5 when mature elements of the liver are forming and before canalicular membrane formation at embryonic days 17 and $18 .^{32,33}$ PCR showed successful deletion of integrin $\beta 1$ in the liver (Figure 1A).

Livers in hsd-itgb1-t and control littermates without the transgene were grossly similar at birth and at 4 weeks of age (data not shown), and there was no difference in the ratio of
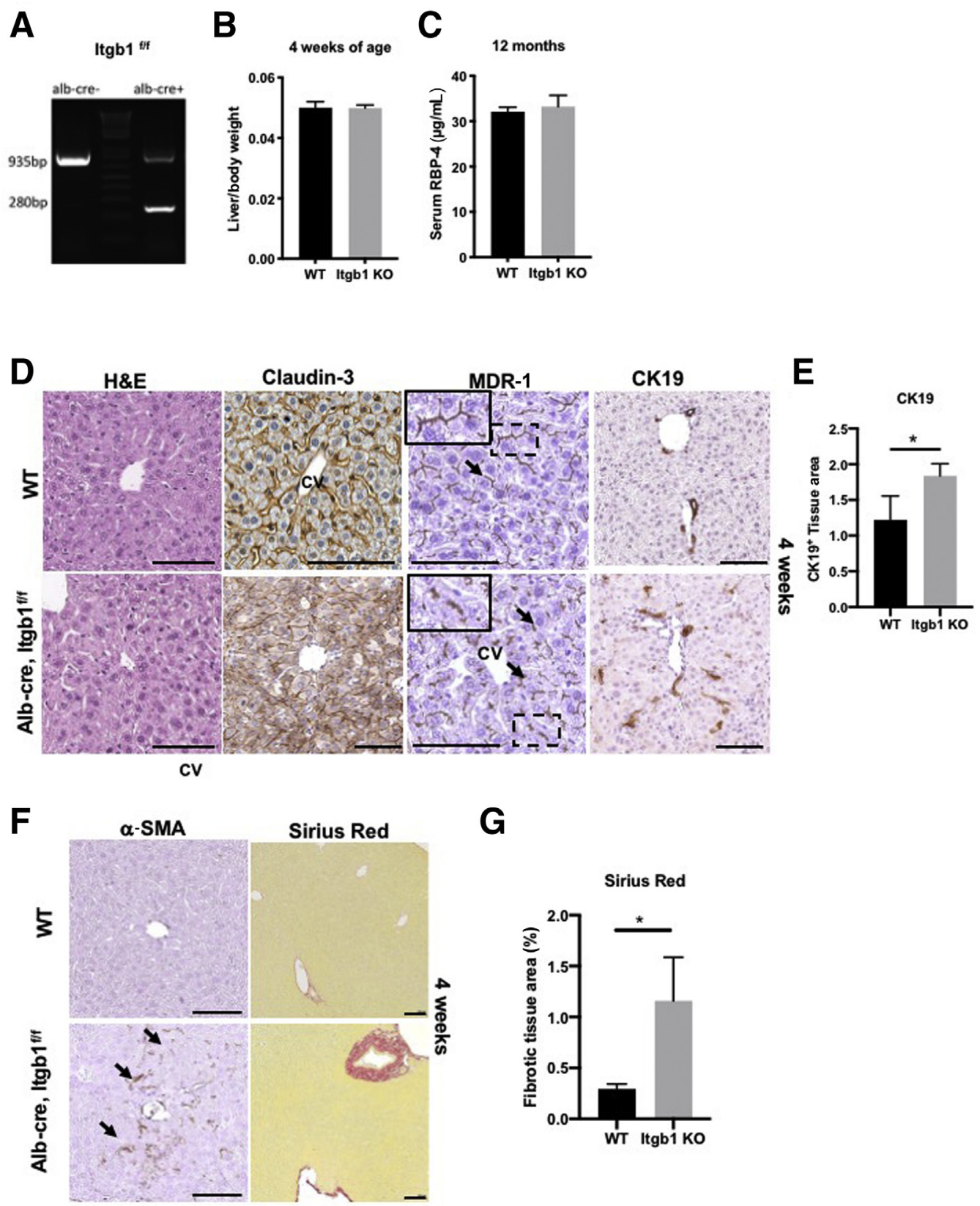

G

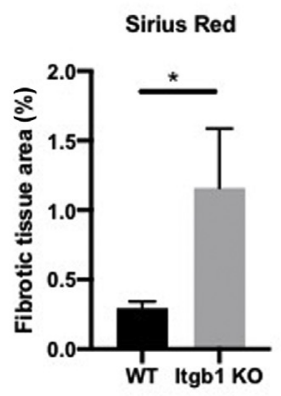

Figure 1 Integrin $\beta 1$ is essential for microscopic patterning of the liver. A: PCR shows successful deletion of integrin $\beta 1$ in the liver as evidenced by the 280 bp fragment. B: The liver to body weight ratio remains unaffected by the deletion at 4 weeks of age. C: Retinol-binding protein 4 (RBP4) levels are normal in hsd-itgb1-t mice at 1 year of age. D: Wild-type (WT) mice at 4 weeks of age display uniformly sized hepatocytes organized in a regular pattern across the lobule without fibrosis. In contrast, hsd-itgb1-t mice contain hepatocytes that vary in size and are arranged irregularly. Claudin-3 staining shows collapse of canalicular double membrane pattern in hsd-itgb1-t livers compared with control. Multidrug resistance transporter 1 (MDR-1) staining shows truncated and irregular canaliculi formation in hsd-itgb1-t mice (black arrows). Dashed boxes are shown at higher magnification in the insets. Cholangiocyte staining with CK19 shows a ductular reaction in hsd-itgb1-t livers with expansion of CK19-positive cells in the liver lobules. E: Morphometry confirms increase in CK19 compared with controls. F: Stellate cells are activated as evidenced by $\alpha$-smooth muscle actin ( $\alpha$-SMA) staining (black arrows) and bile ducts massively fibrose as shown by Sirius Red staining in hsd-itgb1-t livers at 4 weeks of age. G: Quantitation by Sirius Red confirms fibrosis in hsd-itgb1-t livers compared with controls. ${ }^{*} P<0.05$. Scale bars $=100 \mu \mathrm{m}(\mathbf{D}$ and $\mathbf{F})$. CV, central vein; H\&E, hematoxylin and eosin; K0, knockout. 
liver to body weight (Figure 1B). Retinol-binding protein 4, a sensitive marker of liver synthetic function, was similar between wild-type and hsd-itgb1-t mice even at 12 months of age (Figure 1C), as were alanine aminotransferase (18.3 versus $38 \mathrm{U} / \mathrm{L})$, alkaline phosphatase (75.3 versus $53.3 \mathrm{U} / \mathrm{L})$, total bilirubin $(0.23$ versus $0.2 \mathrm{mg} / \mathrm{dL})$, albumin ( 3.26 versus $3.2 \mathrm{~g} / \mathrm{L}$ ), and bile acid ( 1 versus $<1 \mu \mathrm{mol} / \mathrm{L}$ ) levels. These results suggest that integrin $\beta 1$ is not required for gross patterning, growth, or establishing the macroscopic architecture of the liver.

Conversely, microscopy revealed marked disorganization in hsd-itgb1-t mice. According to hematoxylin and eosin and cell membrane claudin-3 staining, control mice displayed uniformly-sized hepatocytes organized in typical single-cell plates across the lobule. In contrast, hsd-itgb1-t mice did not have discernable plates, and hepatocytes varied in size and were arranged irregularly (Figure 1D). Normal tight junction spacing and a double track pattern between adjacent hepatocytes were obliterated in the hsd-itgb1-t mice.

Staining for MDR-1, an apical membrane transporter, exhibited short and irregularly branched canaliculi in hsditgb1-t livers compared with control. Similarly, whereas in normal liver, cholangiocyte staining with CK19 showed formed bile ducts in portal triads, hsd-itgb1-t livers displayed a ductular reaction with expansion of CK19-positive cells in the liver lobules, confirmed by morphometry (Figure 1, D and E). Because ductular reaction correlates with fibrosis, hepatic stellate cells were activated in hsditgb1-t, as evidenced by staining for $\alpha$-smooth muscle actin (Figure 1F). hsd-itgb1-t livers developed significant spontaneous fibrosis by 4 weeks of age around the bile ducts, as assessed by Sirius Red staining and tissue morphometry (Figure 1G). Histologic changes progressed to parenchymal fibrosis by 4 months of age (Supplemental Figure S1).

These findings suggest that integrin $\beta 1$ plays an essential role in organizing the microscopic architecture of the liver and preventing fibrosis.

\section{Integrin- $\beta 1$ Is Essential for Bile Canaliculi Formation in Developing Liver}

To determine the temporal development of these microstructural changes, developing liver in hsd-itgb1-t was examined at earlier time points. The albumin promoter led to cre-recombinase expression around embryonic day 14.5 in hepatoblasts, the time when mature elements of the liver are forming, resulting in loss of integrin $\beta 1$ in both progeny hepatocytes and cholangiocytes, before the development of canalicular membranes. Both hematoxylin and eosin and MDR-1 staining were similar in hsd-itgb1-t compared with control at E15.5 and P1. By 2 weeks of age, however, hsditgb1-t livers exhibited marked alteration of canalicular structure with long, hyperbranched chains indicating that integrin $\beta 1$ is critical for the early development of normal canalicular structure (Figure 2). The relatively normal
MDR-1 staining at P1 compared with the development of canalicular membranes at embryonic days 17 and 18 suggests that integrin $\beta 1$ is essential for the maturation, but not specification, of these elements.

\section{Integrin $\beta 1$ Is Essential to Re-Establish Liver Architecture during Liver Regeneration}

We next hypothesized that integrin $\beta 1$ is essential to reestablish microscopic architecture after injury in adult liver. Hepatectomy was chosen as a "clean" injury with no damage or inflammation in the regenerating regions, allowing separation of the effects of hepatocyte death and inflammation on the development of fibrosis. To perform these experiments, floxed integrin $\beta 1$ mice were infected with AAV8-cre virus. These hsd-itgb1-v mice, over a period of 2 weeks' incubation, did not acutely exhibit altered liver microstructure (data not shown). At the doses used, the virus infects $100 \%$ of hepatocytes but does not injure them or cause inflammation. ${ }^{26}$

After confirming recombination, a two-thirds hepatectomy was performed. Consistent with our hypothesis and results in the developmental hsd-itgb1-t, hsd-itgb1-v mice also developed disordered architecture 3 weeks after surgery compared with control mice. In hsd-itgb1-v livers, histologic examination showed randomly arranged hepatocyte plates and profound architectural disorder, and TUNEL staining revealed scattered hepatocyte apoptosis (Figure 3A). Hsd-itgb1-v livers stained with CK-19 exhibited ductular proliferation with expansion of CK19-positive cells compared with controls, and Sirius Red staining and collagen I immunostaining revealed extensive fibrosis (Figure 3A). These findings were in contrast to control livers, which recreated normal architecture without fibrosis 3 weeks after hepatectomy.

As in hsd-itgb1-t livers, hsd-itgb1-v liver after hepatectomy showed disordered bile canaliculi that were starshaped and displayed abnormal branches as shown by MDR-1 staining (Figure 3B). These post-hepatectomy microstructural findings mirrored the results in the developing livers. The ratio of liver to body weight was significantly decreased at 24 hours after the partial hepatectomy in hsd-itgb1-v mice compared with control mice (Figure 3C) but not at 72 hours, 7 days, or 21 days following partial hepatectomy (Supplemental Figure S2). Serum analyses showed no significant differences in liver function at 24 or 72 hours after surgery (Supplemental Figure S2).

To determine the effect of integrin $\beta 1$ on establishing cell-cell junctions during liver regeneration, immunohistochemistry was performed after partial hepatectomy for gap and cell-cell junction proteins. In hsd-itgb1-v livers compared with control, claudin-3 staining revealed disordered hepatocyte plates, with irregularly sized hepatocytes. The zonation of the hepatic lobule was also disrupted as evident by E-cadherin staining (Figure 3B). To assess if hepatocyte integrin $\beta 1$ loss alters hepatocyte-hepatocyte 
A

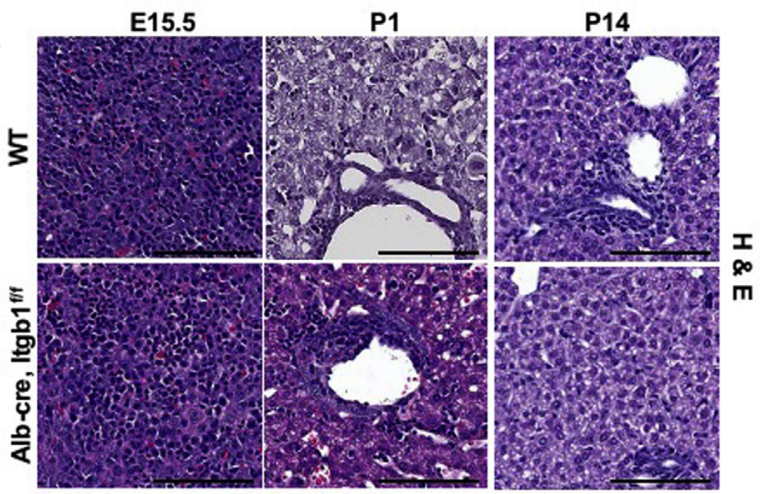

B

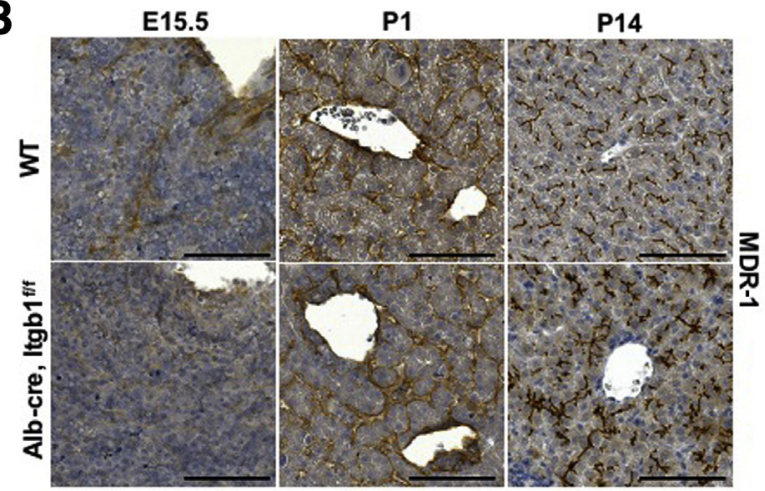

Figure 2 Loss of integrin $\beta 1$ leads to disordered hepatocyte architecture by 14 days' postconception. A: Hematoxylin and eosin (H\&E) staining shows similar hepatocyte architecture in hsd-itgb1-t mice versus controls at $\mathrm{e} 15.5$ and P1 and P14. B: Multidrug resistance transporter 1 (MDR-1) staining shows abnormal branching patterns in hsd-itgb1-t at P14 with extended thick chains compared with control livers. Scale bars $=100$ $\mu \mathrm{m}$ (A and $\mathbf{B})$. WT, wild type.

junctions, liver sections were analyzed by electron microscopy, and massive expansion of cell-cell borders in hsd-itgb1-v livers compared with controls was found (Figure 3D). These results suggest that integrin $\beta 1$ is essential in recreating proper zonation and re-establishing canalicular structure in the liver during regeneration.

\section{Integrin- $\beta 1$ Is Essential to Inhibit TGF- $\beta 1$ Signaling from Hepatocytes during Liver Maturation}

The fibrogenic mechanism caused by loss of integrin $\beta 1$ in hepatocytes was examined next. TGF- $\beta 1$ was a focus because it is a key driver of fibrogenesis. TGF- $\beta 1 \mathrm{mRNA}$ (Figure $4 \mathrm{~A}$ ) and circulating protein in serum were increased in hsd-itgb1-t mice (Figure 4B). TGF- $\beta 1$ protein (Figure 4C) was significantly increased in whole liver as measured by real-time PCR and Western blot compared with controls.

To determine the effect of these changes in TGF- $\beta$ signaling on various signal transduction pathways, Western blot was used to examine mediators of the canonical (SMAD) and non-canonical TGF- $\beta$ signaling pathway in both hsd-itgb1-t mice and hsd-itgb1-v mice. In hsd-itgb1-t mice (Figure 4C), SMAD phosphorylation was unchanged with the deletion of integrin $\beta 1$ compared with control mice, whereas phosphorylation of JNK and ERK1/2 was higher in hsd-itgb1-t mice compared with control mice. However, expression of pP38, pAkt, or pSMAD3 was similar, indicating that these noncanonical TGF- $\beta$ signaling ERK and JNK pathways were unaffected in hsd-itgb1-t mice. To localize these proteins, immunohistochemistry was performed for phospho-ERK1/2, revealing widespread expression in the hsd-itgb1-t mice (Figure 4D). After hepatectomy, hsd-itgb1-v mice did not exhibit significantly different patterns of phosphorylation of ERK or JNK compared with control mice (Supplemental Figure S3).

These findings suggest that loss of integrin $\beta 1$ in hepatocytes increases noncanonical TGF- $\beta$ signaling via JNK and ERK pathways.

\section{ECM Remodeling during Liver Maturation}

To determine how loss of integrin $\beta 1$ influences ECMmodifying enzymes, expression of MMP-13, TIMP-1, and TIMP-2 was examined at 2 weeks of age by Western blot in hsd-itgb1-t mice. Liver protein expression of MMP-13 was significantly lower and TIMP-1 was significantly higher in the liver of hsd-itgb1-t mice compared with control mice. These findings all point to the development of a profibrotic environment with the loss of integrin $\beta 1$.

\section{Integrin $\beta 1$ Specifies Hepatocyte Morphology,} Microscopic Architecture, and Interactions with Stellate Cells

Further mechanistic studies to determine the role of integrin $\beta 1$ on hepatocytes and their relationship to other cells were performed in vitro. Sandwich cultures reproduce hepatocyte microscopic structure and canalicular development, and allow precise manipulations of the cells. ${ }^{34}$

Primary sandwich cultures showed that loss of integrin $\beta 1$ led to hepatocytes that were irregularly shaped and unable to form normal canaliculi. Phase contrast images showed typical canalicular formation in control mice with long thin connections outlining hepatocytes (Figure 5A). In contrast, integrin $\beta 1$-deleted hepatocytes exhibited abnormal, lobulated canaliculi. Phalloidin staining confirmed this pattern (Figure 5A) (confirmed by ZO1 staining, data not shown). These results were similar to the in vivo findings.

The ability of integrin $\beta 1-$ deleted hepatocytes to activate stellate cells was examined next. Conditioned media derived from hepatocytes lacking integrin $\beta 1$ but not controls activated hepatic stellate cells, as illustrated by phenotypic transition with long filaments stained by smooth muscle actin (Figure 5B). This was also confirmed in conditioned media experiments with the hepatic stellate cell line JS1. JS1 cells incubated with conditioned media from hsd-itgb1t-deleted primary hepatocytes revealed an increase in 

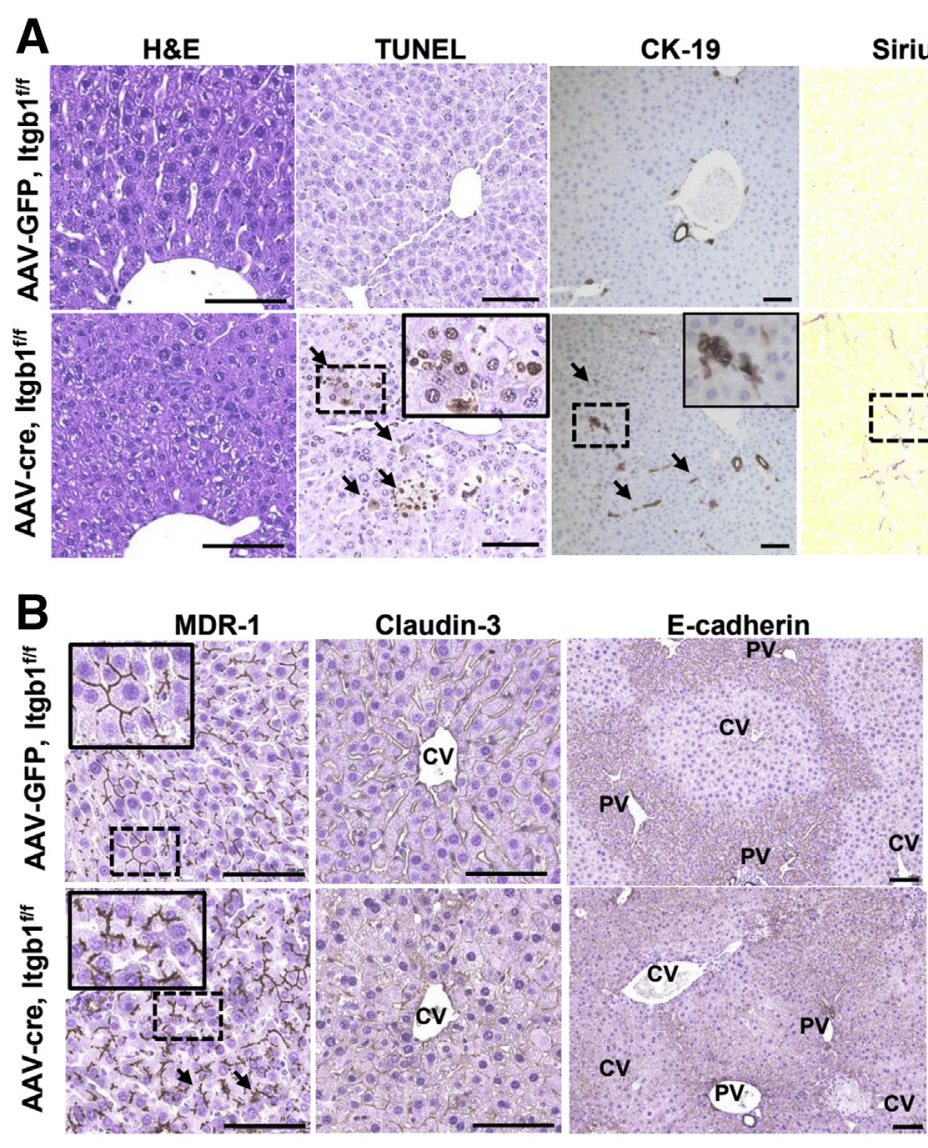

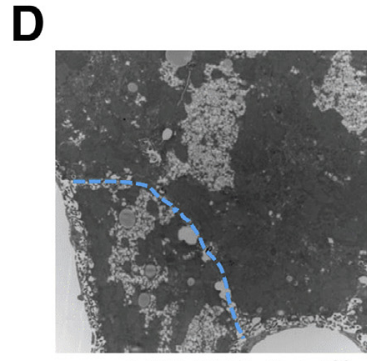

AAV-GFP, Itgb1/f/

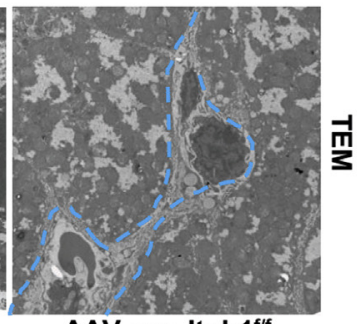

AAV-cre, Itgb1 $1^{\text {tff }}$
C
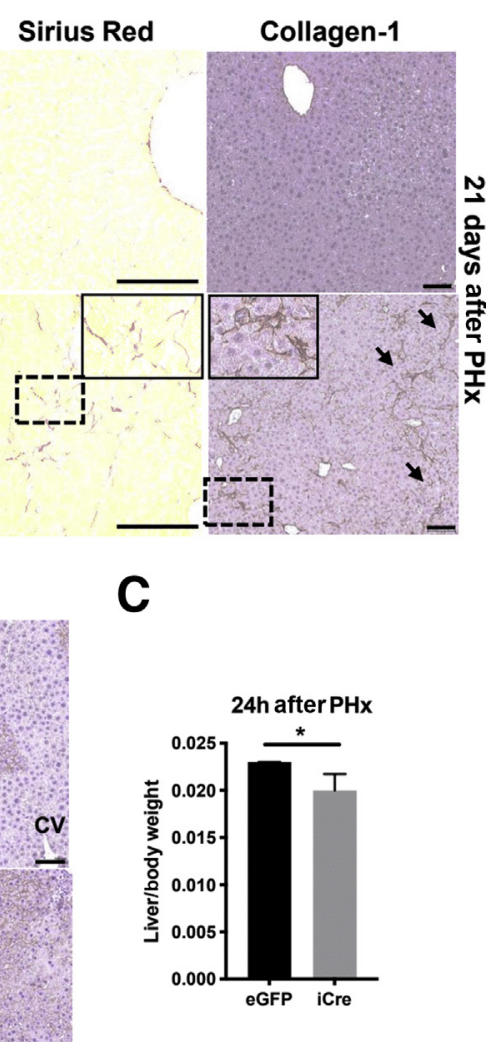

Figure 3 Integrin $\beta 1$ is essential to re-establish liver architecture after injury. A: hsd-itgb1-v mice develop disordered architecture 3 weeks after partial hepatectomy. Hematoxylin and eosin (H\&E) staining shows absent sinusoids and profound architectural disorder in the hsd-itgb1-t mice. TUNEL staining reveals scattered hepatocyte apoptosis in hsd-itgb1-v mice (black arrows). CK19 shows ductular reaction (black arrows) in hsd-itgb1-t mice. Sirius Red staining reveals extensive fibrosis. Immunostaining for collagen I shows no deposition in control mice compared with significant deposition in hsd-itgb1-v mice (black arrows). B: Canaliculi are disordered and star-shaped in hsd-itgb1-v mice by MDR-1 expression. Claudin-3 staining shows disordered canalicular formation with collapse of paired membrane appearance, and E-cadherin expression shows a loss of zonation in hsd-itgb1-v livers. Dashed boxes are shown at higher magnification in the insets. C: Twenty-four hours after hepatectomy (PHx), hsd-itgb1-v livers are smaller than control livers. D: Electron microscopy demonstrates wide cell-cell borders (blue dashed lines) in hsd-itgb1-v livers. ${ }^{*} P<0.05$. Scale bars $=100 \mu \mathrm{m}(\mathbf{A}$ and $\mathbf{B})$. CV, central vein; eGFP, enhanced green fluorescent protein; PV, portal vein.

collagen $1 \alpha 1$ and a trend toward increased $\alpha$-smooth muscle actin mRNA compared with cells incubated with conditioned media from control primary hepatocytes (Figure 5C).

To determine the mechanism of this effect, conditioned media from integrin $\beta 1$-deleted hepatocytes versus control were tested for the presence of TGF- $\beta 1$. Loss of integrin $\beta 1$ doubled the concentration of TGF- $\beta 1$ in the conditioned media compared with controls (Figure 5D). TGF- $\beta 1$ was undetectable in nonconditioned media (data not shown).

\section{Discussion}

A fundamental question of liver biology is how microstructure is determined and maintained. The results presented here identify integrin $\beta 1$ as essential for a myriad of microarchitectural features of the liver, including hepatocyte size, shape, and polarity, and development of cell-cell junctions and canalicular structure. Embryonic studies suggest this effect likely involves the maturation of elements and not their 
A
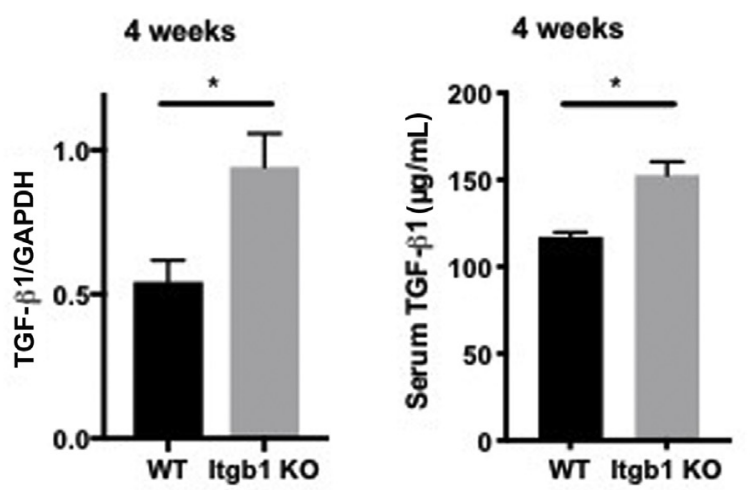

C

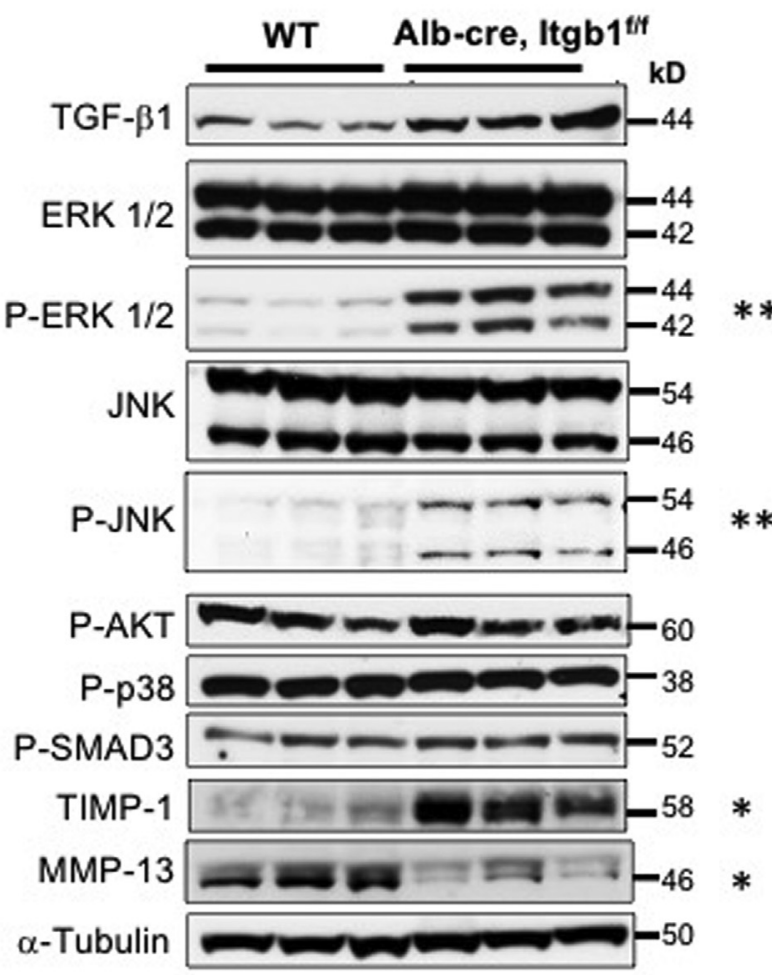

D

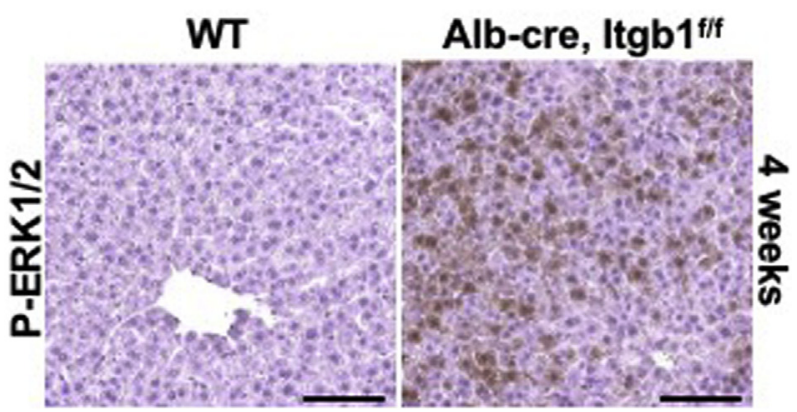

specification. This function is critical both during development and during repair after injury. Importantly, loss of integrin $\beta 1$ results in TGF- $\beta$ secretion that in turn leads to stellate cell activation and fibrosis.

These findings establish an essential role for integrins in the development of liver microstructure and its recreation after injury. The results suggest that local interactions between hepatocytes and ECM provide essential information to establish a global structure and confirm that loss of microstructure leads to a profibrotic state in which TGF- $\beta$ is secreted by hepatocytes to induce stellate cells to lay down collagen.

Whether the pleomorphic effects of deletion of integrin $\beta 1$ are the result of one central defect or many independent ones has not been determined. For example, altering hepatocyte shape in the liver may lead to downstream effects on polarity, cell-cell junctions, and subsequently, TGF- $\beta$ signaling. Alternatively, integrin $\beta 1$ may be performing multiple independent functions related to its anchoring or intracellular messenger functioning.

Multiple lines of evidence support a connection between integrin $\beta 1$ and TGF- $\beta$ in the liver. Loss of integrin $\beta 1$ leads to increased TGF- $\beta$ mRNA in the liver parenchyma as well as protein in the serum. Activation of the Erk pathway is required for TGF- $\beta$-induced events in many systems, and our findings that Erk 1/2 phosphorylation is dramatically upregulated in integrin $\beta 1$-deleted livers strongly supports that TGF- $\beta$ signaling is up-regulated not only in stellate cells, but also in hepatocytes in these mice. ${ }^{35-37}$ Activation of non-canonical JNK pathways is further evidence that TGF- $\beta$ signaling is up-regulated. ${ }^{38,39}$ Previous in vitro studies show that primary hepatocyte cultures using integrin $\beta 1$-deleted hepatocytes fail to fully phosphorylate Erk in response to hepatocyte growth factor challenge. ${ }^{16}$ Our studies examine a more chronic state and are consistent with the profibrotic state for which multiple lines of evidence are presented.

Deletion of integrin $\beta 1$ has a profound effect on matrixmodifying proteins, including TIMP-1 and MMP-13, both known to be active in human liver. Decreases in MMP-13 and increases in its inhibitor TIMP-1 produce a highly profibrotic state. ${ }^{40}$ This result is consistent with our findings

Figure 4 Integrin $\beta 1$ is essential to inhibit transforming growth factor$\beta 1$ (TGF- $\beta 1$ ) signaling from hepatocytes during liver maturation. A: mRNA expression of Tgf- $\beta 1$ was increased in hsd-itgb1-t mice. B: Serum expression of TGF- $\beta 1$ was increased in hsd-itgb1-t mice at 4 weeks of age. C: Western blot showed increased TGF- $\beta 1$, as well as c-Jun N-terminal kinase (JNK) and extracellular signal-regulated kinase 1/2 (ERK1/2) phosphorylation in hsd-itgb1-t mice. There was no significant difference in expression of pAkt and pSMAD3, and pP38. Liver protein expression of tissue inhibitor of metalloproteinase-1 (TIMP-1) was significantly higher in hsd-itgb1-t mice compared with control mice, and matrix metalloproteinase-13 (MMP-13) was significantly lower in hsd-itgb1-t mice compared with control mice. D: After hepatectomy, $\mathrm{p}$-ERK1/2 was widely expressed in hsnitgb1-v livers compared with control livers. $n=5$ WT (A and B); $n=4$ Itgb1 K0 (A and $\mathbf{B}) .{ }^{*} P<0.05,{ }^{*} P>0.01$. Scale bars $=100 \mu \mathrm{m}$. $\mathrm{KO}$, knockout; WT, wild type. 
A

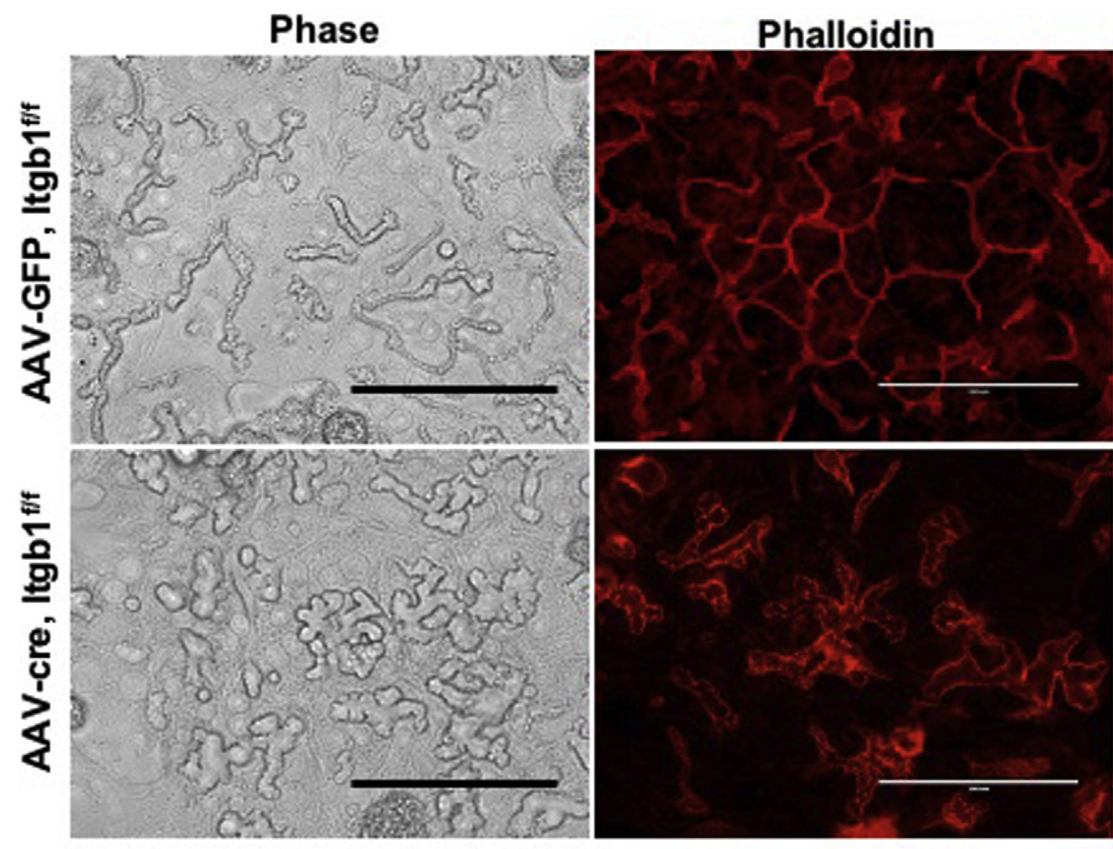

B

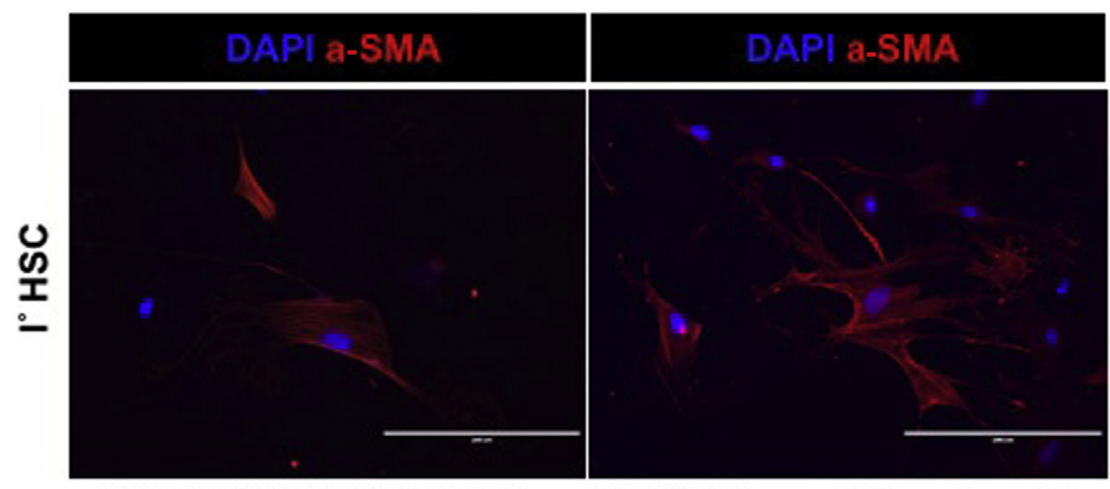

WT conditioned media Itgb1 KO conditioned media

C

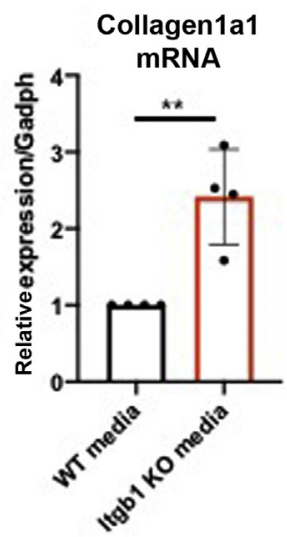

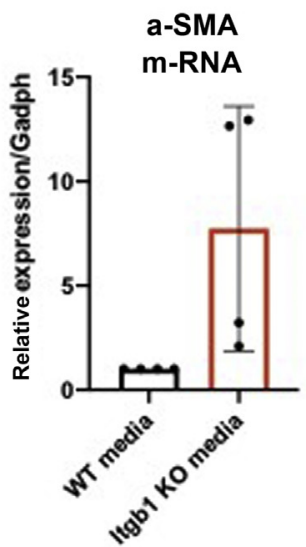

$\mathbf{D}$

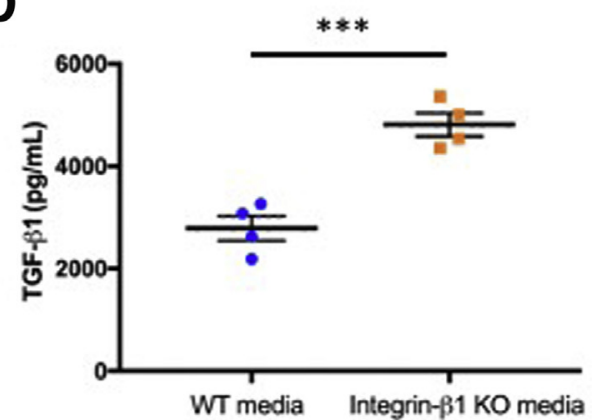

Figure 5 Integrin $\beta 1$ specifies hepatocyte morphology, microscopic architecture, and interactions with stellate cells. A: In primary sandwich cultures, hsd-itgb1-t hepatocytes were irregularly shaped and unable to form normal canaliculi. Phase contrast images show typical canalicular formation in control mice with long thin connections outlining hepatocytes. In contrast, hsd-itgb1-t hepatocytes show abnormal, lobulated canaliculi. Phalloidin staining confirmed that hsd-itgb1-t hepatocytes had abnormal, lobulated canaliculi. B: Conditioned media from hsd-itgb1-t hepatocytes versus controls activated stellate cells, as illustrated by phenotypic transition with long filaments stained by $\alpha$-smooth muscle actin ( $\alpha$-SMA). C: Collagen 1a1 and $\alpha$-SMA was increased in stellate cell culture by conditioned media from hsn-itgb1-v compared with control media. D: Conditioned media from hsd-itgb1-t contained double the concentration of transforming growth factor- $\beta 1$ (TGF- $\beta 1$ ) compared with controls. ${ }^{* *} P<0.01,{ }^{* *} P<0.001$. Scale bars: $100 \mu \mathrm{m}$ (A); $200 \mu \mathrm{m}$ (B). K0, knockout; WT, wild type. Itgb Integrin beta 1. 
of a critical role for integrin $\beta 1$ in preventing the development of fibrosis. Other reports show that fibrosis in patients with hepatitis $C$ is associated with increased integrin $\beta 1$ expression. ${ }^{17} \mathrm{We}$ reconcile these findings by proposing that fibrosis is a final common pathway for a general category of disordered integrin-ECM interactions, which occur both in our system and in human disease.

Our findings that hepatocytes themselves express TGF- $\beta$ when lacking integrin $\beta 1$ establish a potential mechanism for the multiple downstream effects observed. It is tempting to speculate that loss of orientation or positional information in hepatocytes begins a process of fibrosis driven entirely by hepatocytes and suggests that disruption of the hepatocyte-ECM interaction may be sufficient to cause liver fibrosis. This hypothesis could help explain the differential responses of the liver to injury (eg, nonfibrotic healing after massive necrosis following acetaminophen ingestion versus fibrosis after chronic injury as a consequence of the damage to hepatocyte-ECM interaction).

\section{Supplemental Data}

Supplemental material for this article can be found at http://doi.org/10.1016/j.ajpath.2020.10.011.

\section{References}

1. Hynes RO: Integrins: bidirectional, allosteric signaling machines. Cell 2002, 110:673-687

2. Miranti CK, Brugge JS: Sensing the environment: a historical perspective on integrin signal transduction. Nat Cell Biol 2002, 4: E83-E90

3. Gullberg D, Terracio L, Borg TK, Rubin K: Identification of integrinlike matrix receptors with affinity for interstitial collagens. J Biol Chem 1989, 264:12686-12694

4. Johansson S: Demonstration of high affinity fibronectin receptors on rat hepatocytes in suspension. J Biol Chem 1985, 260:1557-1561

5. Forsberg E, Paulsson M, Timpl R, Johansson S: Characterization of a laminin receptor on rat hepatocytes. J Biol Chem 1990, 265: 6376-6381

6. Arias-Salgado EG, Lizano S, Sarkar S, Brugge JS, Ginsberg MH, Shattil SJ: Src kinase activation by direct interaction with the integrin beta cytoplasmic domain. Proc Natl Acad Sci U S A 2003, 100: $13298-13302$

7. Legate KR, Montañez E, Kudlacek O, Fässler R: ILK, PINCH and parvin: the tIPP of integrin signalling. Nat Rev Mol Cell Biol 2006, 7: $20-31$

8. Deakin NO, Turner CE: Paxillin comes of age. J Cell Sci 2008, 121: $2435-2444$

9. Volpes R, van den Oord JJ, Desmet VJ: Distribution of the VLA family of integrins in normal and pathological human liver tissue. Gastroenterology 1991, 101:200-206

10. Wang A, Patrone L, McDonald JA, Sheppard D: Expression of the integrin subunit alpha 9 in the murine embryo. Dev Dyn 1995, 204: 421-431

11. Scoazec JY: Expression of cell-matrix adhesion molecules in the liver and their modulation during fibrosis. J Hepatol 1995, 22:20-27

12. Menon B, Sudhakaran PR: Actin binds to the cytoplasmic tail of alpha 1 subunit of integrin in hepatocytes. Indian J Biochem Biophys 2000, 37:81-85
13. Gohlke H, Schmitz B, Sommerfeld A, Reinehr R, Häussinger D: 5 [beta]1-integrins are sensors for tauroursodeoxycholic acid in hepatocytes. Hepatology 2013, 57:1117-1129

14. Urtasun R, Lopategi A, George J, Leung TM, Lu Y, Wang X, Ge X, Fiel MI, Nieto N: Osteopontin, an oxidant stress sensitive cytokine, up-regulates collagen-I via integrin [alpha](V)[beta](3) engagement and PI3K/pAkt/NF[kappa]B signaling. Hepatology 2012, 55: 594-608

15. Gkretsi V, Apte U, Mars WM, Bowen WC, Luo JH, Yang Y, Yu YP, Orr A, St-Arnaud R, Dedhar S, Kaestner KH, Wu C, Michalopoulos GK: Liver-specific ablation of integrin-linked kinase in mice results in abnormal histology, enhanced cell proliferation, and hepatomegaly. Hepatology 2008, 48:1932-1941

16. Speicher T, Siegenthaler B, Bogorad RL, Ruppert R, Petzold T, Padrissa-Altes S, Bachofner M, Anderson DG, Koteliansky V, Fässler R, Werner S: Knockdown and knockout of [beta]1-integrin in hepatocytes impairs liver regeneration through inhibition of growth factor signalling. Nat Commun 2014, 5:3862

17. Nejjari M, Couvelard A, Mosnier JF, Moreau A, Feldmann G, Degott C, Marcellin P, Scoazec JY: Integrin up-regulation in chronic liver disease: relationship with inflammation and fibrosis in chronic hepatitis C. J Pathol 2001, 195:473-481

18. Henderson NC, Sheppard D: Integrin-mediated regulation of TGF [beta] in fibrosis. Biochem Biophys Acta 2013, 1832:891-896

19. Knittel T, Mehde M, Kobold D, Saile B, Dinter C, Ramadori G: Expression patterns of matrix metalloproteinases and their inhibitors in parenchymal and non-parenchymal cells of rat liver: regulation by TNF-alpha and TGF-beta1. J Hepatol 1999, 30: $48-60$

20. Ikeda K, Wakahara T, Wang YQ, Kadoya H, Kawada N, Kaneda K: In vitro migratory potential of rat quiescent hepatic stellate cells and its augmentation by cell activation. Hepatology 1999, 29:1760-1767

21. Hemmann S, Graf J, Roderfeld M, Roeb E: Expression of MMPs and TIMPs in liver fibrosis - a systematic review with special emphasis on anti-fibrotic strategies. J Hepatol 2007, 46:955-975

22. Iredale JP: Hepatic stellate cell behavior during resolution of liver injury. Semin Liver Dis 2001, 21:427-436

23. Roeb E, Graeve L, Müllberg J, Matern S, Rose-John S: TIMP-1 protein expression is stimulated by IL-1 beta and IL-6 in primary rat hepatocytes. FEBS Lett 1994, 349:45-49

24. Roeb E, Rose-John S, Erren A, Edwards DR, Matern S, Graeve L, Heinrich PC: Tissue inhibitor of metalloproteinases-2 (TIMP-2) in rat liver cells is increased by lipopolysaccharide and prostaglandin E2. FEBS Lett 1995, 357:33-36

25. Raghavan S, Bauer C, Mundschau G, Li Q, Fuchs E: Conditional ablation of betal integrin in skin. Severe defects in epidermal proliferation, basement membrane formation, and hair follicle invagination. J Cell Biol 2001, 150:1149-1160

26. Ho KJ, Bass CE, Kroemer AH, Ma C, Terwilliger E, Karp SJ: Optimized adeno-associated virus 8 produces hepatocyte-specific Cre-mediated recombination without toxicity or affecting liver regeneration. Am J Physiol Gastrointest Liver Physiol 2008, 295: G412-G419

27. Committee for the Update of the Guide for the Care and Use of Laboratory Animals: National Research Council: Guide for the Care and Use of Laboratory Animals: Eighth Edition. Washington, DC, National Academies Press, 2011

28. Junqueira LC, Bignolas G, Brentani RR: Picrosirius staining plus polarization microscopy, a specific method for collagen detection in tissue sections. Histochem J 1979, 11:447-455

29. Masuzaki R, Zhao S, Valerius MT, Tsugawa D, Oya Y, Ray KC, Karp SJ: SOCS2 balances metabolic and restorative requirements during liver regeneration. J Biol Chem 2016, 291: $3346-3358$

30. Weiskirchen R, Gressner AM: Isolation and culture of hepatic stellate cells. Methods Mol Med 2005, 117:99-113 
31. Guo J, Hong F, Loke J, Yea S, Lim CL, Lee U, Mann DA, Walsh MJ, Sninsky JJ, Friedman SL: A DDX5 S480A polymorphism is associated with increased transcription of fibrogenic genes in hepatic stellate cells. J Biol Chem 2010, 285:5428-5437

32. Postic C, Shiota M, Niswender KD, Jetton TL, Chen Y, Moates JM, Shelton KD, Lindner J, Cherrington AD, Magnuson MA: Dual roles for glucokinase in glucose homeostasis as determined by liver and pancreatic beta cell-specific gene knock-outs using Cre recombinase. J Biol Chem 1999, 274:305-315

33. Tanimizu N, Kaneko K, Itoh T, Ichinohe N, Ishii M, Mizuguchi T, Hirata K, Miyajima A, Mitaka T: Intrahepatic bile ducts are developed through formation of homogeneous continuous luminal network and its dynamic rearrangement in mice. Hepatology 2016, 64: $175-188$

34. Swift B, Pfeifer ND, Brouwer KL: Sandwich-cultured hepatocytes: an in vitro model to evaluate hepatobiliary transporter-based drug interactions and hepatotoxicity. Drug Metab Rev 2010, 42: $446-471$
35. Xie L, Law BK, Chytil AM, Brown KA, Aakre ME, Moses HL: Activation of the Erk pathway is required for TGF-beta1-induced EMT in vitro. Neoplasia 2004, 6:603-610

36. Lee MK, Pardoux C, Hall MC, Lee PS, Warburton D, Qing J, Smith SM, Derynck R: TGF-beta activates Erk MAP kinase signalling through direct phosphorylation of ShcA. EMBO J 2007, 26:3957-3967

37. Hough C, Radu M, Doré JJ: Tgf-beta induced Erk phosphorylation of smad linker region regulates smad signaling. PLoS One 2012, 7: e42513

38. Hammaker DR, Boyle DL, Inoue T, Firestein GS: Regulation of the JNK pathway by TGF-beta activated kinase 1 in rheumatoid arthritis synoviocytes. Arthritis Res Ther 2007, 9:R57

39. Zhang YE: Non-Smad pathways in TGF-beta signaling. Cell Res 2009, 19:128-139

40. Mittal R, Patel AP, Debs LH, Nguyen D, Patel K, Grati M, Mittal J, Yan D, Chapagain P, Liu XZ: Intricate functions of matrix metalloproteinases in physiological and pathological conditions. J Cell Physiol 2016, 231:2599-2621 